\title{
CONFORMAL AND CR MAPPINGS ON CARNOT GROUPS
}

\author{
MICHAEL G. COWLING, JI LI, ALESSANDRO OTTAZZI, AND QINGYAN WU \\ (Communicated by Jeremy Tyson)
}

\begin{abstract}
We consider a class of stratified groups with a CR structure and a compatible control distance. For these Lie groups we show that the space of conformal maps coincide with the space of CR and anti-CR diffeomorphisms. Furthermore, we prove that on products of such groups, all CR and anti-CR maps are product maps, up to a permutation isomorphism, and affine in each component. As examples, we consider free groups on two generators, and show that these admit very simple polynomial embeddings in $\mathbb{C}^{N}$ that induce their CR structure.
\end{abstract}

\section{INTRODUCTION}

In this article, we consider the interplay between metric and complex geometry on some model manifolds. This is the first outcome of a larger project which aims to develop a unified theory of conformal and CR structures on the one hand, and to define explicit polynomial embeddings of certain $\mathrm{CR}$ manifolds into $\mathbb{C}^{n}$ on the other. This kind of embedding is what permits the detailed analysis of the $\partial_{b}$ operator carried out by Stein and his collaborators since the 1970s (see [6]). The analogy between CR and conformal geometry is well documented in the case of CR manifolds of hypersurface-type, see, e.g., 7,10 14,17. The easiest and perhaps the most studied example in this setting is that of the Heisenberg group, taken with its sub-Riemannian structure. Here we will focus on those CR manifolds that are stratified groups and that admit a control metric compatible with the CR structure in a suitable sense.

The class of stratified groups that we consider have a particular algebraic structure, which we call tight. These are the indecomposable examples that mimic the Heisenberg group, in the sense that the metric and algebraic structures are very closely tied together. It turns out that the only tight stratified groups are either Heisenberg groups or groups whose Lie algebras have two generators (Corollary 3.2). Tight groups may be endowed with an abstract CR structure. We will show that

Received by the editors November 1, 2019, and, in revised form, March 13, 2020.

2010 Mathematics Subject Classification. Primary 22E25; Secondary 30L10, 32V15, 35R03, $53 \mathrm{C} 23$.

Key words and phrases. Carnot groups, CR mappings, quasiconformal mappings, conformal mappings.

The first and third authors were supported by the Australian Research Council, through grant DP170103025.

The second author was supported by the Australian Research Council, through grant DP 170101060 .

The fourth author was supported by the Natural Science Foundation of China, through Grants 11671185 and 11701250, and by the Natural Science Foundation of Shandong Province, through Grants ZR2018LA002 and ZR2019YQ04.

(C) 2020 by the authors under Creative Commons Attribution-Noncommercial 3.0 License (CC BY NC 3.0) 
the space of $\mathrm{CR}$ and anti-CR automorphisms coincides with the space of conformal maps with respect to a compatible control metric (Theorem 3.3). Hence, we consider the problem of realising our spaces as embedded manifolds. The fact that these groups embed as CR submanifolds of $\mathbb{C}^{n}$ for appropriate $n$ is a consequence of [1] see also [9]. We will find explicit embeddings in the cases of free Lie groups with two generators and step at most 8 (Theorem 4.1). Further, we will show that, on products of tight groups, all CR maps are product maps, up to a permutation isomorphism, and are affine in each component, that is, the composition of a translation with a group automorphism (Theorem 5.5). In order to achieve this, we first show that differentiable quasiconformal mappings on product stratified groups are product mappings, up to an automorphic permutation (Theorem 5.1). The last result is a minor variation of [19, Theorem 1.1].

Here is what follows. In Section 2, we establish the definitions and properties of Carnot groups and conformal mappings that will be used throughout this paper. In Section 3, we introduce CR structures on stratified groups and define a compatible metric when the groups are tight. In this section we prove one of our main results, Theorem 3.3. and we ask whether we can see these CR groups as boundaries of domains in some $\mathbb{C}^{n}$. This is equivalent to constructing explicit embeddings in some $\mathbb{C}^{n}$, which is in turn equivalent to solving a system of differential equations. In Section 4 we find the explicit embeddings for the case of free nilpotent groups with two generators up to step 8 , by solving the differential equations of the previous section. Finally, in Section [5.5. we prove our result about product groups, Theorem 5.5] which is a consequence of Theorem 3.3 and Corollary 5.3 .

\section{Preliminaries}

In this section, we define stratified Lie algebras and Lie groups, and show how to put sub-Riemannian structures on these. We also define the Pansu derivative and consider quasiconformal mappings for these structures.

2.1. Stratified Lie algebras. A Lie algebra $\mathfrak{g}$ is said to be stratified of step $\ell$ if

$$
\mathfrak{g}=\mathfrak{g}_{-1} \oplus \cdots \oplus \mathfrak{g}_{-\ell},
$$

where $\left[\mathfrak{g}_{-j}, \mathfrak{g}_{-1}\right]=\mathfrak{g}_{-j-1}$ when $1 \leq j \leq \ell$, while $\mathfrak{g}_{-\ell} \neq\{0\}$ and $\mathfrak{g}_{-\ell-1}=\{0\}$; this implies that $\mathfrak{g}$ is nilpotent. We assume that $\operatorname{dim}(\mathfrak{g})$ is at least 3 to avoid degenerate cases.

We write $\pi_{j}$ for the canonical projection of $\mathfrak{g}$ onto $\mathfrak{g}_{-j}, \mathfrak{Z}(\mathfrak{g})$ for the centre of $\mathfrak{g}$, and $\operatorname{Aut}(\mathfrak{g})$ for the group of automorphisms of $\mathfrak{g}$. In particular, for each $s \in \mathbb{R}^{+}$, the dilation $\delta_{s} \in \operatorname{Aut}(\mathfrak{g})$ is defined to be $\sum_{j=1}^{\ell} s^{j} \pi_{j}$.

For a linear map of $\mathfrak{g}$, preserving all the subspaces $\mathfrak{g}_{-j}$ of the stratification is equivalent to commuting with dilations, and to having a block-diagonal matrix representation. We call such maps strata-preserving. We write $\operatorname{Aut}^{\delta}(\mathfrak{g})$ for the subset of Aut $(\mathfrak{g})$ of strata-preserving automorphisms; these are determined by their action on $\mathfrak{g}_{-1}$. A stratified Lie algebra $\mathfrak{g}$ is said to be totally nonabelian if $\mathfrak{g}_{-1} \cap$ $\mathfrak{Z}(\mathfrak{g})=\{0\}$. If $\mathfrak{g}$ is totally nonabelian, then $\mathfrak{g}$ has a finest direct sum decomposition (see [5, Theorem 2.3]):

$$
\mathfrak{g}=\bigoplus_{k=1}^{K} \mathfrak{g}^{k}
$$


where the $\mathfrak{g}^{k}$ are nontrivial totally nonabelian stratified Lie algebras that commute pairwise, with the additional property that, given any direct sum decomposition $\bigoplus_{l=1}^{L} \tilde{\mathfrak{g}}^{l}$ of $\mathfrak{g}$ into ideals, the set $\{1, \ldots, K\}$ may be partitioned into disjoint subsets $J_{1}, \ldots, J_{L}$ such that

$$
\tilde{\mathfrak{g}}^{l}=\bigoplus_{j \in J_{l}} \mathfrak{g}^{j} \quad \forall l \in\{1, \ldots, L\} .
$$

When $j, k \in\{1, \ldots, K\}$, we write $j \sim k$ if and only if there is a strata-preserving isomorphism from $\mathfrak{g}^{j}$ to $\mathfrak{g}^{k}$; then $\sim$ is an equivalence relation. For each equivalence class $[j]$ and each $k \in[j]$, choose a stratified Lie algebra $\mathfrak{g}^{[j]}$ isomorphic to $\mathfrak{g}^{j}$, and a strata-preserving isomorphism $I^{k}$ from $\mathfrak{g}^{[i]}$ to $\mathfrak{g}^{k}$, whose inverse we write as $I^{-k}$.

When $\sigma$ lies in $S_{m}^{\sim}$, the group of permutations of $\{1, \ldots, m\}$ that preserve the equivalence classes of $\sim$, define $I^{\sigma} \in \operatorname{Aut}^{\delta}(\mathfrak{g})$ by first setting

$$
I^{\sigma}(X)=I^{\sigma(j)} I^{-j}(X)
$$

for all $X \in \mathfrak{g}^{j}$ and all $j \in\{1, \ldots, m\}$, and then extending this definition to $\mathfrak{g}$ by linearity. It is well known and easy to check that the map $\sigma \mapsto I^{\sigma}$ embeds $S_{m}^{\sim}$ in $\operatorname{Aut}^{\delta}(\mathfrak{g})$. We denote the image by $\operatorname{Perm}(\mathfrak{g})$.

2.2. Stratified Lie groups. Let $G$ be a stratified Lie group of step $\ell$. This means that $G$ is connected and simply connected, and its Lie algebra $\mathfrak{g}$ is stratified with $\ell$ layers. The identity of $G$ is written $e$

Since $G$ is nilpotent, connected, and simply connected, the exponential map exp is a bijection from $\mathfrak{g}$ to $G$, with inverse log. In particular, if $\left\{U_{j}: j=1, \ldots, n\right\}$ is a basis of $\mathfrak{g}$, we may write every point of $G$ as $\exp \left(u_{1} U_{1}+\cdots+u_{n} U_{n}\right)$, which we denote by $\left(u_{1}, \ldots, u_{n}\right)$ and call exponential coordinates of the first kind. We also write $\delta_{s}$ for the automorphism of $G$ given by $\exp \circ \delta_{s} \circ \log$. The differential $T \mapsto\left(T_{*}\right)_{e}$ is a one-to-one correspondence between automorphisms of $G$ and of $\mathfrak{g}$, and $T=\exp \circ\left(T_{*}\right)_{e} \circ \log$. We denote by $\operatorname{Aut}(G)$ the group of automorphisms of $G$, and by $\operatorname{Aut}^{\delta}(G)$ the subgroup of automorphisms that commute with dilations.

A stratified connected simply connected Lie group $G$ is called totally nonabelian or a direct product if its Lie algebra is totally nonabelian or a Lie algebra direct sum. The finest direct product decomposition of the group is that associated to the finest direct sum decomposition of the Lie algebra.

First, we state and prove a preliminary lemma.

Lemma 2.1. Suppose that $G$ is a simply connected nilpotent Lie group, with an orthonormal basis $\left\{U_{j}: j=1, \ldots, n\right\}$ for its Lie algebra. Let $\sum_{n=0}^{\infty} c_{n} z^{n}$ be the power series of the function $z /\left(1-e^{-z}\right)$ (extended to 0 by continuity), which converges in the ball with centre 0 and radius $2 \pi$. Then the left-invariant vector field $X$ corresponding to $X \in \mathfrak{g}$, evaluated at $\exp (Y)$ in $G$, is given in exponential coordinates of the first kind by

$$
X_{\exp Y}=\left\langle\left(\sum_{k=0}^{\infty} c_{k} \operatorname{ad}^{k}(Y)\right) X, U_{j}\right\rangle \partial_{u_{j}},
$$

where the sum terminates when $k$ is sufficiently large as $\operatorname{ad}(Y)$ is nilpotent.

Proof. The derivative of the exponential map exp at $Y \in \mathfrak{g}$ is given by

$$
\frac{1-\exp (-\operatorname{ad}(Y))}{\operatorname{ad}(Y)}=\sum_{k=0}^{\infty} \frac{(-1)^{k}}{(k+1) !} \operatorname{ad}^{k}(Y)
$$


(see, for example, [15, Theorem 2.14.3]), and the series terminates because $G$ is nilpotent.

The coefficients $c_{j}$ may be determined inductively from the condition

$$
\left(\sum_{k=0}^{\infty} \frac{(-1)^{k}}{(k+1) !} z^{k}\right)\left(\sum_{k=0}^{\infty} c_{k} z^{k}\right)=1,
$$

and we find that

$$
c_{0}=1, \quad c_{1}=\frac{1}{2}, \quad c_{2}=\frac{1}{12}, \quad c_{3}=0, \quad c_{4}=\frac{-1}{720}, \quad \ldots
$$

In a stratified group $G$, more can be said. We say that a function $f$ on $G$ is homogeneous of degree $d$ if $f\left(\delta_{s} x\right)=s^{d} f(x)$, and a differential operator $D$ on $G$ is homogeneous of degree $e$ (which may be negative) if $D f$ is homogeneous of degree $d+e$ whenever $f$ is homogeneous of degree $d$. We write $\operatorname{deg}(f)$ and $\operatorname{deg}(D)$ for these degrees.

If $X \in \mathfrak{g}_{-k}$, then the associated vector field $X$ is homogeneous of degree $-k$. If we take a basis $\left\{U_{j}: j=1, \ldots, n\right\}$ of $\mathfrak{g}$, where each $U_{j}$ belongs to some $\mathfrak{g}_{-d(j)}$, and use exponential coordinates of the first kind on $G$, that is, we write

$$
\left(u_{1}, \ldots, u_{n}\right):=\exp \left(u_{1} U_{1}+\ldots u_{n} U_{n}\right),
$$

then the coordinate function $u_{j}$ is homogeneous of degree $d(j)$. A vector field that is homogeneous of degree $d$ is a linear combination of the left-invariant vector fields $U_{1}, \ldots, U_{n}$, with coefficients $c_{j}$ that are homogeneous functions, and $d=$ $\operatorname{deg}\left(c_{j}\right)+\operatorname{deg}\left(U_{j}\right)$.

2.3. The Pansu differential. We denote by $L_{p}$ the left translation by $p$ in $G$, that is, $L_{p} q=p q$ for all $q \in G$. The subbundle $H G$ of the tangent bundle $T G$ given by $H_{p} G=\left(L_{p}\right)_{*}\left(\mathfrak{g}_{-1}\right)$ is called the horizontal distribution. We write $\Omega$ for an arbitrary nonempty connected open subset of $G$. The differential of a differentiable map $f: \Omega \rightarrow G$ is written $f_{*}$. We recall that a continuous map $f: \Omega \rightarrow G$ is Pansu differentiable at $p \in \Omega$ if the limit

$$
\lim _{s \rightarrow 0+} \delta_{s}^{-1} \circ L_{f(p)}^{-1} \circ f \circ L_{p} \circ \delta_{s}(q)
$$

converges, uniformly for $q$ in compact subsets of $G$, to a strata-preserving homomorphism of $G$, written $D f_{p}(q)$. If $f$ is Pansu differentiable at $p$, then $\log \circ D f_{p} \circ \exp$ is a Lie algebra homomorphism, written $d f_{p}$, and

$$
d f_{p}(X)=\lim _{s \rightarrow 0+} \log \circ \delta_{s}^{-1} \circ L_{f(p)}^{-1} \circ f \circ L_{p} \circ \delta_{t} \circ \exp (X)
$$

exists, uniformly for $X$ in compact subsets of $\mathfrak{g}$. We call $D f_{p}$ the Pansu derivative and $d f_{p}$ the Pansu differential of $f$ at $p$. By construction, both $D f_{p}$ and $d f_{p}$ commute with dilations, and so in particular, $d f_{p}$ is a strata-preserving Lie algebra homomorphism.

Note that if $T$ is a strata-preserving automorphism of $G$, then its Pansu derivative $D T(p)$ coincides with $T$ at every point, and its Pansu differential $d T(p)$ coincides with the Lie differential $\log \circ T \circ \exp$ at every point. Thus our notation is a little different from the standard Lie theory notation, but is not ambiguous. 
2.4. The sub-Riemannian distance. We fix a scalar product $\langle\cdot, \cdot\rangle$ on $\mathfrak{g}_{-1}$, and we define a left-invariant Riemannian metric on the horizontal distribution by the formula

$$
\langle V, W\rangle_{p}=\left\langle\left(L_{p^{-1}}\right)_{*}(V),\left(L_{p^{-1}}\right)_{*}(W)\right\rangle
$$

for all $V, W \in H_{p} G$ and all $p \in G$. This gives rise to a left-invariant sub-Riemannian or Carnot-Carathéodory distance function $\varrho$ on $G$. To define this, we first say that a smooth curve $\gamma$ is horizontal if $\dot{\gamma}(t) \in H_{\gamma(t)} G$ for every $t$. Then we define the distance $\varrho(p, q)$ between points $p$ and $q$ by

$$
\varrho(p, q):=\inf \int_{0}^{1}\left(\langle\dot{\gamma}(t), \dot{\gamma}(t)\rangle_{\gamma(t)}\right)^{1 / 2} d t
$$

where in the infimum we take all horizontal curves $\gamma:[0,1] \rightarrow G$ such that $\gamma(0)=p$ and $\gamma(1)=q$. The distance function is homogeneous, symmetric, and left-invariant, that is,

$$
s^{-1} \varrho\left(\delta_{s} p, \delta_{s} q\right)=\varrho(p, q)=\varrho(q, p)=\varrho(r q, r p) \quad \forall p, q, r \in G \quad \forall s \in \mathbb{R}^{+} ;
$$

in particular, $\varrho(p, q)=\varrho\left(q^{-1} p, e\right)$. The stratified group $G$, equipped with the distance $\varrho$, is known as a Carnot group.

2.5. Quasiconformal automorphisms and maps. We write $S(V)$ for the unit sphere in a normed vector space $V$.

Suppose that $\lambda \geq 1$. We say that $T \in \operatorname{Aut}^{\delta}(\mathfrak{g})$ is $\lambda$-quasiconformal if and only if

$$
\max \left\{\|T X\|: X \in S\left(\mathfrak{g}_{-1}\right)\right\} \leq \lambda \min \left\{\|T X\|: X \in S\left(\mathfrak{g}_{-1}\right)\right\} .
$$

Of course, every $T \in \operatorname{Aut}^{\delta}(\mathfrak{g})$ is $\lambda$-quasiconformal for sufficiently large $\lambda$.

Suppose that $s \in \mathbb{R}^{+}$. In a Carnot group, the distortion $H(f, p, s)$ of a map $f: \Omega \rightarrow G$ at a point $p \in \Omega$ and at scale $s \in \mathbb{R}^{+}$is defined by

$$
H(f, p, s)=\frac{\sup \{\varrho(f(x), f(p)): x \in \Omega, \varrho(x, p) \leq s\}}{\inf \{\varrho(f(x), f(p)): x \in \Omega, \varrho(x, p) \geq s\}} .
$$

The map $f$ is $\lambda$-quasiconformal in $\Omega$ if

$$
\limsup _{s \rightarrow 0+} H(f, p, s) \leq \lambda \quad \forall p \in \Omega,
$$

and $f$ is quasiconformal if it is $\lambda$-quasiconformal for some $\lambda \in \mathbb{R}^{+}$.

If the map $f$ is $C^{1}$, then it is $\lambda$-quasiconformal in $\Omega$ if and only if its Pansu differential $d f_{p}$ is $\lambda$-quasiconformal at all $p \in \Omega$. It is known that 1-quasiconformal maps on Carnot groups and on some sub-Riemannian manifolds are smooth (see [2,3]); such maps are also known as conformal maps.

\section{CR STRATIFIED GROUPS AND CARNot GROUPS}

In this section, we consider CR structures on stratified groups and Carnot groups; we consider an example with an illustrious history, and construct many new examples of Carnot groups as boundaries of domains. 
3.1. CR stratified groups. Let $G$ be a stratified group such that $\operatorname{dim} \mathfrak{g}_{-1}=2 m$ and let $n$ be the integer such that $2 m+n=\operatorname{dim} G$. We define an almost complex structure on $\mathfrak{g}_{-1}$ to be a linear isomorphism $J: \mathfrak{g}_{-1} \rightarrow \mathfrak{g}_{-1}$ such that $J^{2}=-$ Id and

$$
[X, Y]=[J X, J Y] \quad \text { and } \quad[X, J Y]=-[J X, Y]
$$

for all $X, Y \in \mathfrak{g}_{-1}$. A stratified group $G$ equipped with such a mapping $J$ is said to be a $C R$ stratified group of type $(m, n)$. Let $L=\operatorname{span}\left\{X-i J X: X \in \mathfrak{g}_{-1}\right\}$. It is easy to check that (3.1) is equivalent to $L$ being abelian in the complexification $\mathfrak{g}_{\mathbb{C}}$ of $\mathfrak{g}$.

We say that $T \in \operatorname{Aut}^{\delta}(\mathfrak{g})$ is a $C R$ automorphism or an anti-CR automorphism if

$$
\left.T\right|_{\mathfrak{g}_{-1}} J=\left.J T\right|_{\mathfrak{g}_{-1}} \quad \text { or }\left.\quad T\right|_{\mathfrak{g}_{-1}} J=-\left.J T\right|_{\mathfrak{g}_{-1}}
$$

equivalently, $T_{\mathbb{C}}(L) \subseteq L$ or $\overline{T_{\mathbb{C}}}(L) \subseteq L$, where $T_{\mathbb{C}}$ denotes the linear extension of $T$ to $\mathfrak{g}_{\mathbb{C}}$. Notice that the inverse of a CR automorphism is also a CR automorphism. A diffeomorphism $f: \Omega \rightarrow \Upsilon$ between domains in $G$ is a $C R$ mapping or an anti$C R$ mapping if and only if its Pansu differential $d f_{p}$ is a CR automorphism or an anti-CR automorphism for every $p \in \Omega_{1}$. In this section we will study the structure and the $\mathrm{CR}$ diffeomorphisms of $\mathrm{CR}$ stratified groups. In particular, we will show that, for a class of these groups, the space of conformal maps with respect to a compatible metric coincides with the space of CR maps. Last but not least, we will show some explicit embeddings of $\mathrm{CR}$ stratified groups into $\mathbb{C}^{m+n}$ via a $\mathrm{CR}$ diffeomorphism.

3.2. Tight groups. We say that a stratified group is tight if $\mathfrak{g}$ is totally nonabelian, its finest direct product decomposition has only one factor, and $\operatorname{dim} \mathfrak{g}_{-2}=1$. Equivalently,

where

$$
\mathfrak{g}_{-1}=\operatorname{span}\left\{X_{1}, \ldots, X_{m}, Y_{1}, \ldots, Y_{m}\right\}
$$

$$
\left[X_{j}, X_{l}\right]=\left[Y_{j}, Y_{l}\right]=0 \quad \text { and } \quad\left[X_{j}, Y_{l}\right]=\delta_{j l} U \quad \forall j, l=1, \ldots, m .
$$

It is straightforward to check that the space $L$, defined by

$$
L:=\operatorname{span}\left\{X_{j}-i Y_{j}: j=1, \ldots, m\right\},
$$

satisfies $[L, L]=\{0\}$. So tight stratified groups are CR with respect to the almost complex structure determined by the requirements that $J X_{j}=Y_{j}$ and $J Y_{j}=-X_{j}$ for every $j=1, \ldots, m$.

Lemma 3.1. Let $G$ be a tight $C R$ stratified group. If $m>1$, then $U$ is central, that is, $\left[X_{j}, U\right]=\left[Y_{j}, U\right]=0$ when $j=1, \ldots, m$.

Proof. We argue by contradiction. Suppose that $\left[X_{j}, U\right] \neq 0$ for some $j$. Renumbering if necessary, we may assume that $j=1$. Then $\left[X_{1},\left[X_{2}, Y_{2}\right]\right] \neq 0$. However, by the Jacobi identity and (3.2),

$$
\left[X_{1},\left[X_{2}, Y_{2}\right]\right]=\left[\left[X_{1}, X_{2}\right], Y_{2}\right]+\left[X_{2},\left[X_{1}, Y_{2}\right]\right]=0
$$

which gives a contradiction. We may show that $\left[Y_{j}, U\right]=0$ similarly.

Corollary 3.2. Let $G$ be a tight $C R$ stratified group with $\operatorname{dim}\left(\mathfrak{g}_{-1}\right)=2 m$. Then exactly one of the following holds:

(i) $\mathfrak{g}_{-1}=\operatorname{span}\left\{X_{1}, Y_{1}\right\}$ and $\mathfrak{g}_{-3} \neq\{0\}$, 
(ii) $\mathfrak{g}_{-1}=\operatorname{span}\left\{X_{1}, \ldots, X_{m}, Y_{1}, \ldots, Y_{m}\right\}, \mathfrak{g}_{-2}=\operatorname{span}\{U\}$ and $\mathfrak{g}_{-3}=\{0\}$. In this case $\mathfrak{g}$ is the Heisenberg algebra of dimension $2 m+1$.

When $G$ is tight, we consider the element $U^{*} \in \mathfrak{g}^{*}$ uniquely defined by $U^{*}(U)=1$ and $U^{*}(X)=0$ for all $X \in \bigoplus_{k \neq 2} \mathfrak{g}_{-k}$, and the left-invariant one-form $\theta$ such that $\theta_{e}=U^{*}$. Then the bilinear form

$$
B_{\theta}(X, Y)=d \theta(X, J Y)
$$

is a scalar product on $\mathfrak{g}_{-1}$ for which $\left\{X_{1}, \ldots, X_{m}, Y_{1}, \ldots, Y_{m}\right\}$ is an orthonormal basis. Moreover, $B_{\theta}$ is compatible with $J$, in the sense that

$$
B_{\theta}(J X, J Y)=B_{\theta}(X, Y) \quad \forall X, Y \in \mathfrak{g}_{-1} .
$$

We define a Carnot group structure on $G$ using the left-invariant metric on the horizontal subbundle that coincides with $B_{\theta}$ at the identity.

Theorem 3.3. Let $G$ be a tight stratified group with the Carnot distance determined by $B_{\theta}$. Let $f: \Omega \rightarrow G$ be a homeomorphism from a connected open subset $\Omega$ of $G$ onto its image. Then $f$ is 1-quasiconformal if and only if $f$ is $C R$ or anti-CR.

Proof. We say that $T \in \operatorname{Aut}^{\delta}(\mathfrak{g})$ is conformal if

$$
\|T(X)\|=\lambda\|X\| \quad \forall X \in \mathfrak{g}_{-1},
$$

or, equivalently, if $T^{t} T=\lambda^{2} \mathrm{Id}$ for some $\lambda \in \mathbb{R}_{+}$. Here $T^{t}$ denotes the transpose with respect to $B_{\theta}$. It is well known that $f$ is 1-quasiconformal if and only if $d f_{p}$ is conformal for every $p \in \Omega[3$. Therefore, it is enough to show that every conformal automorphism is a CR or anti-CR automorphism, and vice versa. Since $G$ is tight, either $\operatorname{dim} \mathfrak{g}_{-1}=2$ or $G$ is the Heisenberg group of dimension $2 m+1$. It is straightforward to show in both cases that for all $T \in \operatorname{Aut}^{\delta}(\mathfrak{g})$, the condition $\left.\left.T^{t}\right|_{\mathfrak{g}_{-1}} T\right|_{\mathfrak{g}_{-1}}=\lambda$ Id is equivalent to $\left.T\right|_{\mathfrak{g}_{-1}} J=\left.J T\right|_{\mathfrak{g}_{-1}}$ or $\left.T\right|_{\mathfrak{g}_{-1}} J=-\left.J T\right|_{\mathfrak{g}_{-1}}$.

The theorem above holds for every left-invariant metric that is compatible with the CR structure. Indeed, let $B^{\prime}$ be any scalar product on $\mathfrak{g}_{-1}$ with the property that

$$
B^{\prime}(J X, J Y)=B^{\prime}(X, Y) \quad \forall X, Y \in \mathfrak{g}_{-1} .
$$

Then there is $A \in G L(2 \ell, \mathbb{R})$ such that $B^{\prime}(X, Y)=B_{\theta}(A X, A Y)$. The compatibility condition and the definition of $A$ imply that $B_{\theta}(J A X, J A Y)=B_{\theta}(A J X, A J Y)$, which in turn implies that $A J=J A$ or $A J=-J A$. Therefore $A$ induces a CR or anti-CR automorphism of $\mathfrak{g}$, and so by Theorem 3.3 the left-invariant metrics with respect to $B_{\theta}$ and $B^{\prime}$ are conformally equivalent.

3.3. CR embeddings of tight groups. We are now deriving a system of equations whose solutions, if they exist, yield explicit CR embeddings of tight groups $G$ into $\mathbb{C}^{m+n}$ that generalise those considered by Nagel and Stein; our embeddings are more closely related to work of Andreotti and Hill [1, and generalise work of the fourth-named author and her collaborators [16, 17]. As the Heisenberg groups are well understood, we concentrate on the case where $\operatorname{dim} \mathfrak{g}_{-1}=2$ and $\mathfrak{g}_{-3}$ is nontrivial. Our construction involves several steps.

First, extend an orthonormal basis $\{X, Y\}$ of $\mathfrak{g}_{-1}$ to a basis $\left\{X, Y, U_{1}, \ldots, U_{n}\right\}$ of $\mathfrak{g}$, where we choose the $U_{j}$ from the iterated commutators of $X$ and $Y$ in order to first span $\mathfrak{g}_{-2}$, then $\mathfrak{g}_{-3}$, and so on. We then extend the inner product on $\mathfrak{g}_{-1}$ 
to an inner product on $\mathfrak{g}$ so that our basis is orthonormal. We use exponential coordinates of the first kind, and take an element of $G$ to be

$$
\left(x, y, u_{1}, \ldots u_{n}\right):=\exp \left(x X+y Y+u_{1} U_{1}+\cdots+u_{n} U_{n}\right) .
$$

By Lemma 2.1, the left-invariant vector field $T$ corresponding to an element $T$ of $\mathfrak{g}$ is given in these coordinates by

$$
\begin{aligned}
T_{\exp Y} & =\langle T, X\rangle \partial_{x}+\langle T, Y\rangle \partial_{y}+\sum_{j=1}^{n}\left\langle\left(\sum_{k=0}^{\infty} c_{k} \operatorname{ad}^{k}(Y)\right) T, U_{j}\right\rangle \partial_{u_{j}} \\
& =a_{T} \partial_{x}+b_{T} \partial_{y}+\sum_{j=1}^{n} p_{T, j}\left(x, y, u_{1}, \ldots, u_{n}\right) \partial_{u_{j}},
\end{aligned}
$$

where $a_{T}=\langle T, X\rangle, b_{T}=\langle T, Y\rangle$, and $p_{T, j}\left(x, y, u_{1}, \ldots, u_{n}\right)$ is equal to

$$
\sum_{l=1}^{n} \sum_{k=0}^{\infty} c_{k}\left\langle\operatorname{ad}^{k}\left(x X+y Y+u_{1} U_{1}+\cdots+u_{n} U_{n}\right) T, U_{l}\right\rangle \partial_{u_{l}} .
$$

The functions $p_{T, j}$ are polynomials of bounded degree, since the series above has finitely many nonzero terms, and are homogeneous if $T$ is homogeneous. In particular,

$$
X=\partial_{x}+\sum_{j=1}^{n} p_{X, j}\left(x, y, u_{1}, \ldots, u_{n}\right) \partial_{u_{j}}
$$

and

$$
Y=\partial_{y}+\sum_{j=1}^{n} p_{Y, j}\left(x, y, u_{1}, \ldots, u_{n}\right) \partial_{u_{j}} .
$$

Now we seek to map $G$ into the surface

$$
\left\{\left(x, y, u_{1}, \ldots, u_{n}, v_{1}, \ldots, v_{n}\right) \in \mathbb{R}^{2+2 n}: v_{j}=q_{j}\left(x, y, u_{1}, \ldots, u_{n}\right), j=1, \ldots, n\right\},
$$

where the $q_{j}$ are homogeneous polynomials of positive degree, using the map $\phi$, defined by taking $\phi\left(x, y, u_{1}, \ldots, u_{n}\right)$ equal to

$$
\left(x, y, u_{1}, \ldots, u_{n}, q_{1}\left(x, y, u_{1}, \ldots, u_{n}\right), \ldots, q_{n}\left(x, y, u_{1}, \ldots, u_{n}\right)\right) .
$$

The map $\phi$ is evidently a $C^{\infty}$ embedding, and 0 lies on the surface. In the obvious extension of our coordinate system, the differential $\phi_{*}$ of $\phi$ satisfies

$$
\phi_{*}(T)=T+\sum_{j=1}^{n}\left(T q_{j}\right) \partial_{v_{j}} .
$$

We identify $\left(x, y, u_{1}, \ldots, u_{n}, v_{1}, \ldots, v_{n}\right) \in \mathbb{R}^{2+2 n}$ with $\left(z, w_{1}, \ldots, w_{n}\right) \in \mathbb{C}^{1+n}$, where $z=x+i y$ and $w_{j}=u_{j}+i v_{j}$. When we do this, our embedding is a CR embedding if and only if the complex $(1,0)$ vector field $Z=X+i Y$ on $G$ maps to a $(1,0)$ vector field tangent to the surface in $\mathbb{C}^{1+n}$. Now

$$
\phi_{*}(Z)=\phi_{*}(X)+i \phi_{*}(Y),
$$

and this is a $(1,0)$ vector field if and only if the coefficient of $\partial_{v_{j}}$ is $i$ times the coefficient of $\partial_{u_{j}}$ for all $j$, that is,

$$
\left(X q_{j}\right)+i\left(Y q_{j}\right)=i\left(p_{X, j}+i p_{Y, j}\right)
$$


or equivalently,

$$
\left\{\begin{array}{l}
X q_{j}=-p_{Y, j}\left(x, y, u_{1}, \ldots, u_{n}\right), \\
Y q_{j}=p_{X, j}\left(x, y, u_{1}, \ldots, u_{n}\right) .
\end{array}\right.
$$

This is a nontrivial system of differential equations, and we do not know whether it can be solved in general. There are certainly many examples where this is possible, for instance, if $\mathfrak{g}$ is filiform-see [16, 18]. In the next section, we will consider the case of free nilpotent Lie groups with two generators and use an alternative coordinate system to solve (3.4) for the case when these Lie groups have step at most 8 .

If the stratified group $G$ is not tight, there is no obvious canonical choice of a compatible Carnot structure. Hence the extent to which we can generalise our study of the interplay between conformal and CR structures in the general case is unclear.

\section{Free nilpotent Lie groups}

In this section we focus on solving the system of equations (3.4) in the tight case, that is, for free nilpotent Lie groups whose Lie algebra has two generators and step at least 2. We introduce some further notation, that in some cases will replace that of the previous sections. Denote by $\mathfrak{f}_{2, s}$ the free nilpotent Lie algebra of step $s$ with two generators, and let $n=\operatorname{dim} \mathfrak{f}_{2, s}$. Recall that $\mathfrak{f}_{2, s}$ is the biggest nilpotent Lie algebra of step $s$ generated by iterated brackets of two generators $X_{1}$ and $X_{2}$. The elements in the linear span of

$$
\left[X_{\alpha_{1}}, \ldots,\left[X_{\alpha_{k-1}}, X_{\alpha_{k}}\right] \ldots\right]
$$

where $X_{\alpha_{1}}, \ldots, X_{\alpha_{k}}$ are vectors in $\mathfrak{f}_{2, s}$, are said to have length at most $k$. We now recall the recursive definition of the Hall basis 8 for $\mathfrak{f}_{2, s}$. Each element in the basis is a monomial in the generators. The generators $X_{1}$ and $X_{2}$ are elements of the basis and of length 1. Assume that we have defined basis elements of lengths $1, \ldots, \ell-1$ and that they are simply ordered in such a way that $X<Y$ if $\operatorname{length}(X)<$ length $(Y)$. If length $(X)=r$ and length $(Y)=t$, and $\ell=r+t$, then $[X, Y]$ is a basis element of length $\ell$ if:

1. $X$ and $Y$ are basis elements and $X>Y$, and

2. if $X=[Z, W]$, then $Y \geq W$.

Number the basis elements using this ordering, i.e., $X_{3}=\left[X_{2}, X_{1}\right], X_{4}=$ $\left[X_{3}, X_{1}\right], X_{5}=\left[X_{3}, X_{2}\right]$, etc. Consider a basis element $X_{i}$ as a bracket in the lower order basis elements, $\left[X_{j_{1}}, X_{k_{1}}\right]$, where $j_{1}>k_{1}$. If we repeat this process with $X_{j_{1}}$, we get $X_{i}=\left[\left[X_{j_{2}}, X_{k_{2}}\right], X_{k_{1}}\right]$, where $k_{2} \leq k_{1}$ by the Hall basis conditions. Continuing in this fashion, we end up with

$$
X_{i}=\left[\left[\ldots\left[\left[X_{2}, X_{i_{1}}\right], X_{i_{2}}\right], \ldots, X_{i_{m-1}}\right], X_{i_{m}}\right],
$$

where $i_{1}=1$ and $i_{\ell} \leq i_{\ell+1}$ when $2 \leq \ell \leq m-1$. Since this expansion involves $m$ brackets, we shall write $d(i)=m$ and define $d(1)=d(2)=0$. This process naturally associates a multi-index $I(i)=\left(a_{1}, \ldots, a_{n}\right)$ to each Hall basis element $X_{i}$, defined by $a_{r}=\#\left\{t: i_{t}=r\right\}$. Note that $I(i)=(0, \ldots, 0)$ for $i=1,2$. Let $x=\left(x_{1}, \ldots, x_{n}\right)$ be coordinates in $\mathbb{R}^{n}$. For every $j \geq 3$, we define the monomial $p_{j}$ 
by

$$
p_{j}(x):=\frac{(-1)^{d(j)}}{I(j) !} x^{I(j)}
$$

where $x^{I(j)}=x_{1}^{I(j)_{1}} \cdots x_{n}^{I(j)_{n}}$ and $I(j) !=I(j)_{1} ! \cdots I(j)_{n} !$, if $I(j)=\left(I(j)_{1}, \ldots, I(j)_{n}\right)$. Notice that $I(j)_{1} \geq 1$. It will be convenient to represent the bracket (4.1) by the vector $\left(2,1, i_{2}, \ldots, i_{m}\right)$. We stress that any one of the formula (4.1) for $X_{i}$, the vector $\left(2,1, i_{2}, \ldots, i_{m}\right)$, or the monomial $p_{i}$, uniquely describes the other two.

The vector fields

$$
X_{1}=\frac{\partial}{\partial x_{1}} \quad \text { and } \quad X_{2}=\frac{\partial}{\partial x_{2}}+\sum_{j \geq 3} p_{j} \frac{\partial}{\partial x_{j}}
$$

generate the Lie algebra $\mathfrak{f}_{2, s}$. We now rewrite (3.4) using these vector fields as generators. Thus, we look for polynomials $q_{j}$ solving

$$
\begin{cases}X_{1} q_{j} & =-p_{j} \\ X_{2} q_{j} & =0\end{cases}
$$

for every $j \geq 3$. Since $p_{j}$ is a monomial, the first equation integrates to

$$
q_{j}=c_{j} x_{1} p_{j}+r_{j},
$$

where $c_{j} \in(0,1]$ and $r_{j}=r_{j}\left(x_{2}, \ldots, x_{n}\right)$. We substitute this in the second equation to obtain

$$
c_{j} x_{1} X_{2} p_{j}+X_{2} r_{j}=c_{j} x_{1}\left(\frac{\partial}{\partial x_{2}} p_{j}+\sum_{k>2} p_{k} \frac{\partial}{\partial x_{k}} p_{j}\right)+\frac{\partial}{\partial x_{2}} r_{j}+\sum_{\ell>2} p_{\ell} \frac{\partial}{\partial x_{\ell}} r_{j}=0 .
$$

Since $I(t)_{1} \geq 1$ when $t \geq 3$, it follows that $\partial r_{j} / \partial x_{2}$ is the only term that does not depend on $x_{1}$, so it is zero, and $r_{j}=r_{j}\left(x_{3}, \ldots, x_{n}\right)$. Hence for a free nilpotent Lie algebra with two generators, the system (3.4) can be solved if we can find $r_{j}\left(x_{3}, \ldots, x_{n}\right)$ such that

$$
c_{j} x_{1}\left(\frac{\partial}{\partial x_{2}} p_{j}+\sum_{k>2} p_{k} \frac{\partial}{\partial x_{k}} p_{j}\right)+\sum_{\ell>2} p_{\ell} \frac{\partial}{\partial x_{\ell}} r_{j}=0
$$

for all $j \geq 3$. We now solve this system of equations for free Lie algebras up to step 8 .

Theorem 4.1. Let $\mathfrak{f}_{2, s}$ be a free nilpotent Lie algebra of step $s$ at most 8 . Then the system of equations (4.2) admits a solution of the form

$$
r_{j}\left(x_{3}, \ldots, x_{n}\right)=\sum_{k>2} a_{k}^{j} x_{k}+\sum_{\ell>2} b_{\ell}^{j} x_{\ell}^{2},
$$

for some $a_{k}^{j}, b_{\ell}^{j} \in \mathbb{R}$. In particular, if $s \leq 5$, then $b_{\ell}^{j}=0$.

Vice versa, if $s \geq 9$, then there is $j \geq 3$ such that $\sum_{k>2} a_{k}^{j} x_{k}+\sum_{\ell>2} b_{\ell}^{j} x_{\ell}^{2}$ does not solve (4.2).

For a free nilpotent Lie algebra with two generators, we may represent the monomials $p_{\ell}$ by the vector $\left(2,1, \ell_{1}, \ldots, \ell_{m}\right)$. We stress that this vector is not the same as $I(\ell)$. Vice versa, in order for such a vector to represent a nonzero monomial $p_{\ell}$, it must be that $\left(2,1, \ell_{1}, \ldots, \ell_{m-1}\right)$ represents a basis vector higher than $X_{\ell_{m}}$ and with $X_{\ell_{m}}>X_{\ell_{m-1}}$. Using these rules, we may easily construct all vectors for 
TABle 1. Monomials up to step 8.

\begin{tabular}{|c|c|c|}
\hline Step & Vectors & Monomials \\
\hline 2 & $(2,1)$ & $p_{3}=-x_{1}$ \\
\hline 3 & $(2,1,1),(2,1,2)$ & $p_{4}=\frac{1}{2} x_{1}^{2}, p_{5}=x_{1} x_{2}$ \\
\hline 4 & $\begin{array}{l}(2,1,1,1),(2,1,1,2) \\
(2,1,2,2)\end{array}$ & $\begin{array}{l}p_{6}=-\frac{1}{6} x_{1}^{3}, p_{7}=-\frac{1}{2} x_{1}^{2} x_{2} \\
p_{8}=-\frac{1}{2} x_{1} x_{2}^{2}\end{array}$ \\
\hline 5 & $\begin{array}{l}(2,1,1,1,1),(2,1,1,1,2) \\
(2,1,1,2,2),(2,1,2,2,2) \\
(2,1,1,3),(2,1,2,3)\end{array}$ & $\begin{array}{l}p_{9}=\frac{1}{24} x_{1}^{4}, p_{10}=\frac{1}{6} x_{1}^{3} x_{2} \\
p_{11}=\frac{1}{4} x_{1}^{2} x_{2}^{2}, p_{12}=\frac{1}{6} x_{1} x_{2}^{3} \\
p_{13}=-\frac{1}{2} x_{1}^{2} x_{3}, p_{14}=-x_{1} x_{2} x_{3}\end{array}$ \\
\hline 6 & $\begin{array}{l}(2,1,1,1,1,1),(2,1,1,1,1,2) \\
(2,1,1,1,2,2),(2,1,1,2,2,2) \\
(2,1,2,2,2,2),(2,1,1,1,3) \\
(2,1,1,2,3),(2,1,2,2,3) \\
(2,1,2,4)\end{array}$ & $\begin{array}{l}p_{15}=-\frac{1}{120} x_{1}^{5}, p_{16}=-\frac{1}{24} x_{1}^{4} x_{2} \\
p_{17}=-\frac{1}{12} x_{1}^{3} x_{2}^{2}, p_{18}=-\frac{1}{12} x_{1}^{2} x_{2}^{3} \\
p_{19}=-\frac{1}{24} x_{1} x_{2}^{4}, p_{20}=\frac{1}{6} x_{1}^{3} x_{3} \\
p_{21}=\frac{1}{2} x_{1}^{2} x_{2} x_{3}, p_{22}=\frac{1}{2} x_{1} x_{2}^{2} x_{3} \\
p_{23}=-x_{1} x_{2} x_{4}\end{array}$ \\
\hline 7 & $\begin{array}{l}(2,1,1,1,1,1,1),(2,1,1,1,1,1,2) \\
(2,1,1,1,1,2,2),(2,1,1,1,2,2,2) \\
(2,1,1,2,2,2,2),(2,1,2,2,2,2,2) \\
(2,1,1,1,1,3),(2,1,1,1,2,3) \\
(2,1,1,2,2,3),(2,1,2,2,2,3) \\
(2,1,1,3,3),(2,1,2,3,3) \\
(2,1,1,1,4),(2,1,1,1,5) \\
(2,1,1,2,4),(2,1,1,2,5) \\
(2,1,2,2,4),(2,1,2,2,5)\end{array}$ & $\begin{array}{l}p_{24}=\frac{1}{720} x_{1}^{6}, p_{25}=\frac{1}{120} x_{1}^{5} x_{2} \\
p_{26}=\frac{1}{48} x_{1}^{4} x_{2}^{2}, p_{27}=\frac{1}{36} x_{1}^{3} x_{2}^{3} \\
p_{28}=\frac{1}{48} x_{1}^{2} x_{2}^{4}, p_{29}=\frac{1}{120} x_{1} x_{2}^{5} \\
p_{30}=-\frac{1}{24} x_{1}^{4} x_{3}, p_{31}=-\frac{1}{6} x_{1}^{3} x_{2} x_{3} \\
p_{32}=-\frac{1}{4} x_{1}^{2} x_{2}^{2} x_{3}, p_{33}=-\frac{1}{6} x_{1} x_{2}^{3} x_{3} \\
p_{34}=\frac{1}{4} x_{1}^{2} x_{3}^{2}, p_{35}=\frac{1}{2} x_{1} x_{2} x_{3}^{2} \\
p_{36}=\frac{1}{6} x_{1}^{3} x_{4}, p_{37}=\frac{1}{6} x_{1}^{3} x_{5} \\
p_{38}=\frac{1}{2} x_{1}^{2} x_{2} x_{4}, p_{39}=\frac{1}{2} x_{1}^{2} x_{2} x_{5} \\
p_{40}=\frac{1}{2} x_{1} x_{2}^{2} x_{4}, p_{33}=\frac{1}{2} x_{1} x_{2}^{2} x_{5}\end{array}$ \\
\hline 8 & $\begin{array}{l}(2,1,1,1,1,1,1,1),(2,1,1,1,1,1,1,2) \\
(2,1,1,1,1,1,2,2),(2,1,1,1,1,2,2,2) \\
(2,1,1,1,2,2,2,2),(2,1,1,2,2,2,2,2) \\
(2,1,2,2,2,2,2,2),(2,1,1,1,1,1,3) \\
(2,1,1,1,1,2,3),(2,1,1,1,2,2,3) \\
(2,1,1,2,2,2,3),(2,1,2,2,2,2,3) \\
(2,1,1,1,3,3),(2,1,1,2,3,3) \\
(2,1,2,2,3,3),(2,1,1,1,1,4) \\
(2,1,1,1,1,5),(2,1,1,1,2,4) \\
(2,1,1,1,2,5),(2,1,1,2,2,4) \\
(2,1,1,2,2,5),(2,1,2,2,2,4) \\
(2,1,2,2,2,5),(2,1,1,3,4) \\
(2,1,1,3,5),(2,1,2,3,4) \\
(2,1,2,3,5),(2,1,1,2,6) \\
(2,1,2,2,6),(2,1,2,2,7)\end{array}$ & $\begin{array}{l}p_{41}=-\frac{1}{5040} x_{1}^{7}, p_{42}=-\frac{1}{720} x_{1}^{6} x_{2} \\
p_{43}=-\frac{1}{240} x_{1}^{5} x_{2}^{2}, p_{44}=-\frac{1}{144} x_{1}^{4} x_{2}^{3} \\
p_{45}=-\frac{1}{14} x_{1}^{3} x_{2}^{4}, p_{46}=-\frac{1}{240} x_{1}^{2} x_{2}^{5} \\
p_{47}=-\frac{1}{720} x_{1} x_{2}^{6}, p_{48}=\frac{1}{120} x_{1}^{5} x_{3} \\
p_{49}=\frac{1}{24} x_{1}^{4} x_{2} x_{3}, p_{50}=\frac{1}{12} x_{1}^{3} x_{2}^{2} x_{3} \\
p_{51}=\frac{1}{12} x_{1}^{2} x_{2}^{3} x_{3}, p_{52}=\frac{1}{24} x_{1} x_{2}^{4} x_{3} \\
p_{53}=-\frac{1}{12} x_{1}^{3} x_{3}^{2}, p_{54}=-\frac{1}{4} x_{1}^{2} x_{2} x_{3}^{2} \\
p_{55}=-\frac{1}{4} x_{1} x_{2}^{2} x_{3}^{2}, p_{56}=\frac{1}{24} x_{1}^{4} x_{4} \\
p_{57}=-\frac{1}{24} x_{1}^{4} x_{5}, p_{58}=-\frac{1}{6} x_{1}^{3} x_{2} x_{4} \\
p_{59}=-\frac{1}{6} x_{1}^{3} x_{2} x_{5}, p_{60}=-\frac{1}{4} x_{1}^{2} x_{2}^{2} x_{4} \\
p_{61}=-\frac{1}{4} x_{1}^{2} x_{2}^{2} x_{5}, p_{62}=-\frac{1}{6} x_{1} x_{2}^{3} x_{4} \\
p_{63}=-\frac{1}{6} x_{1} x_{2}^{3} x_{5}, p_{64}=\frac{1}{2} x_{1}^{2} x_{3} x_{4} \\
p_{65}=\frac{1}{2} x_{1}^{2} x_{3} x_{5}, p_{66}=x_{1} x_{2} x_{3} x_{4} \\
p_{67}=x_{1} x_{2} x_{3} x_{5}, p_{68}=\frac{1}{2} x_{1}^{2} x_{2} x_{6} \\
p_{69}=\frac{1}{2} x_{1} x_{2}^{2} x_{6}, p_{70}=\frac{1}{2} x_{1} x_{2}^{2} x_{7}\end{array}$ \\
\hline
\end{tabular}

a given step. For example, if the step is 2 , we only have the monomial $p_{3}$, corresponding to the vector $(2,1)$. In step 3 , we have to add two more monomials, $p_{4}$, and $p_{5}$, corresponding to the vectors $(2,1,1)$ and $(2,1,2)$. We write all vectors and monomials up to step 8 in Table 1 .

Theorem 4.1 will be a consequence of the following three lemmas.

Lemma 4.2. Let $\mathfrak{f}_{2, s}$ be a free nilpotent Lie algebra of step $s$ at most 8 . Then for all $j, k \geq 3$,

$$
x_{1} p_{k} \frac{\partial}{\partial x_{k}} p_{j} \in \operatorname{span}\left\{p_{\ell}\right\}
$$

for some $\ell=\ell(j, k)$.

Proof. We say that $X_{j}$ has height $h(j)$ if $X_{j} \in \mathfrak{g}_{-h(j)}$. Observe that $p_{j}$ is homogeneous of degree $h(j)-1$. It follows that, if $x_{1} p_{k} \frac{\partial}{\partial x_{k}} p_{j} \neq 0$, then it is homogeneous 
of the same degree as $p_{j}$. If this is the case, for our statement to be true, it must be that $h(\ell)=h(j)$. Moreover, $\frac{\partial}{\partial x_{k}} p_{j}$ can only be nonzero if $3 \leq k \leq 7$. Using these observations, the claim readily follows by inspecting Table 1

Lemma 4.3. Let $\mathfrak{f}_{2, s}$ be a free nilpotent Lie algebra of step $s$ at most 8 . Then for every $j \geq 3$,

$$
x_{1} \frac{\partial}{\partial x_{2}} p_{j} \in \begin{cases}\operatorname{span}\left\{p_{\ell}\right\} & \text { if } j \neq 23, \\ \operatorname{span}\left\{x_{4} p_{4}\right\} & \text { if } j=23, \\ \operatorname{span}\left\{x_{6} p_{6}\right\} & \text { if } j=68, \\ \operatorname{span}\left\{x_{7} p_{7}\right\} & \text { if } j=70\end{cases}
$$

for some $\ell=\ell(j)$. In particular, if $s \leq 5$, then $x_{1} \frac{\partial}{\partial x_{2}} p_{j} \in \operatorname{span}\left\{p_{\ell}\right\}$.

Proof. Given $p_{j}$, we study the action of $x_{1} \frac{\partial}{\partial x_{2}}$ on the associated vector $(2,1$, $\left.j_{2}, \ldots, j_{m}\right)$. If $j_{k} \neq 2$ for all $k$, then $x_{1} \frac{\partial}{\partial x_{2}} p_{j}=0$ and we are done. Otherwise, let $k$ be the smallest integer such that $j_{k}=2$. Then the action of $x_{1} \frac{\partial}{\partial x_{2}}$ replaces the $j_{k}$ th entry with 1 , and more precisely,

$$
\left(2,1, j_{2}, \ldots, j_{m}\right) \mapsto\left(2,1, j_{2}, \ldots, j_{k-1}, 1, j_{k+1}, \ldots, j_{m}\right) .
$$

If $\left(2,1, j_{2}, \ldots, j_{k-1}, 1, j_{k+1}, \ldots, j_{m}\right)$ represents a monomial $p_{\ell}$ for some $\ell$, then we are done. By inspecting Table 11, this always occurs except for the vectors $(2,1,2,4),(2,1,1,2,6)$, and $(2,1,2,2,7)$, which represent the monomials $p_{23}, p_{68}$, and $p_{70}$. In these cases,

$$
x_{1} \frac{\partial}{\partial x_{2}} p_{23}=-x_{1} \frac{\partial}{\partial x_{2}} x_{1} x_{2} x_{4}=-x_{1}^{2} x_{4}=-x_{4} p_{4}
$$

and, similarly, $x_{1} \frac{\partial}{\partial x_{2}} p_{68}=-3 x_{6} p_{6}$ and $x_{1} \frac{\partial}{\partial x_{2}} p_{70}=-2 x_{7} p_{7}$.

Lemma 4.4. Let $\mathfrak{f}_{2, s}$ be a free nilpotent Lie algebra of step 9. Then there is $j$ for which $\sum_{k>2} a_{k}^{j} x_{k}+\sum_{\ell>2} b_{\ell}^{j} x_{\ell}^{2}$ is not a solution of (4.2) for every $a_{k}^{j}, b_{\ell}^{j} \in \mathbb{R}$.

Proof. When $s=9$, consider the monomial $p_{j}=x_{1} x_{2} x_{4} x_{5}$, corresponding to the vector $(2,1,2,4,5)$. A direct computation shows that every solution $r_{j}$ of (4.2) for this monomial contains $c_{j} x_{4}^{2} x_{5}$ for some $c_{j} \neq 0$.

Proof of Theorem 4.1. In view of Lemmas 4.2 and 4.3, if $j \neq 23$ we may rewrite the equation (4.2) as

$$
\sum_{v>2} a_{v} p_{v}+\sum_{\ell>2} p_{\ell} \frac{\partial}{\partial x_{\ell}} r_{j}=\sum_{\ell>2}\left(a_{\ell}+\frac{\partial}{\partial x_{\ell}} r_{j}\right) p_{\ell}=0
$$

for some constants $a_{v}$. Then $r_{j}=-\sum_{\ell>3} a_{\ell} x_{\ell}$ is a solution. If $j=23$, then (4.2) becomes

$$
-c_{4} x_{4} p_{4}+\sum_{v>2, v \neq 4} b_{v} p_{v}+\sum_{\ell>2} p_{\ell} \frac{\partial}{\partial x_{\ell}} r_{23}=0
$$

for some constants $b_{v}$. Hence $r_{23}=-\sum_{v>2, v \neq 4} b_{v} x_{v}+\frac{c_{4}}{2} x_{4}^{2}$ is a solution. A quadratic solution can be found in the same way for $j=68$ and $j=70$.

Theorem 4.1 suggests that all free tight groups can be embedded into $\mathbb{C}^{1+n}$ by means of polynomial functions. The pattern that these polynomials follow is at the moment unknown to the authors. For example, we find linear solutions for groups up to step 5 and then again at step 7 , whereas quadratic solutions are only sufficient 
up to step 8. However, our result gives an algorithm for finding these embeddings for anyone to use and that we plan to implement for MAPLE. Hopefully, this will provide us with enough examples to look again at the global picture.

\section{Products}

We say that a mapping on $G$ is affine if it is the composition of a left translation with an element in $\operatorname{Aut}^{\delta}(G)$.

Theorem 5.1. Suppose that $G$ is a totally nonabelian Carnot group, with finest direct product decomposition $G^{1} \times \cdots \times G^{m}$, where $m>1$. Let $f: G \rightarrow G$ be a $C^{1}$ quasiconformal map. Then $f$ is composed of a group automorphism that permutes the groups $G^{j}$ and a product bi-Lipschitz map.

Proof. We write either $p$ or $\left(p^{1}, \ldots, p^{m}\right)$ for a typical element of $G$.

The Pansu differential of a $C^{1}$ global quasiconformal mapping $f$ is continuous, and hence its $\operatorname{Perm}(\mathfrak{g})$ component is constant. This is an automorphism of $\mathfrak{g}$ and by conjugation with the exponential may be considered as an automorphism of $G$, and is therefore quasiconformal. By composing with the inverse of this automorphism if necessary, we may assume that the Pansu differential $d f_{p}$ of $f$ is a product automorphism (see [5, Corollary 3.4]).

If we take a horizontal curve $\gamma$ in one of the factors $G^{j}$, then $f \circ \gamma$ is again a horizontal curve, whose Pansu derivative is $d f \circ \dot{\gamma}$, and so $f \circ \gamma$ moves in the factor $G^{j}$ and is fixed in the other factors. The groups $G^{j}$ mutually commute, and it follows immediately that $f$ is a product map: we may find maps $f^{j}: G^{j} \rightarrow G^{j}$ such that

$$
f\left(p^{1}, \ldots, p^{m}\right)=\left(f^{1}\left(p^{1}\right), \ldots, f^{m}\left(p^{m}\right)\right) \quad \forall p \in G .
$$

The Pansu differential $d f_{p}$ is also a product map:

$$
d f_{p}=\left(d f_{p^{1}}^{1}, \ldots, d f_{p^{m}}^{m}\right) \quad \forall p \in G
$$

If $f$ is $\lambda$-quasiconformal, it follows immediately that when $j \neq k$,

$$
\max \left\{\left\|d f_{p^{j}}^{j}(X)\right\|: X \in S\left(\mathfrak{g}_{-1}^{j}\right)\right\} \leq \lambda \min \left\{\left\|d f_{p^{k}}^{k}(X)\right\|: X \in S\left(\mathfrak{g}_{-1}^{k}\right)\right\}
$$

for all $p \in G$. Define

$$
c_{k}=\inf \left\{\left\|d f_{p^{k}}^{k}(X)\right\|: X \in S\left(\mathfrak{g}_{-1}^{k}\right), p^{k} \in G^{k}\right\}
$$

for all $k$, and now fix $k$ such that $c_{k}=\min \left\{c_{j}: j \in\{1, \ldots, m\}\right\}$. Then, when $j \neq k$,

$$
\sup \left\{\left\|d f_{p^{j}}^{j}(X)\right\|: X \in S\left(\mathfrak{g}_{-1}^{j}\right), p^{j} \in G^{j}\right\} \leq \lambda c_{k} .
$$

Fix $j$ different from $k$. Since

$$
\begin{aligned}
& \sup \left\{\left\|d f_{p^{k}}^{k}(X)\right\|: X \in S\left(\mathfrak{g}_{-1}^{k}\right), p^{k} \in G^{k}\right\} \\
& \quad \leq \lambda \inf \left\{\left\|d f_{p^{j}}^{j}(X)\right\|: X \in S\left(\mathfrak{g}_{-1}^{j}\right), p^{j} \in G^{j}\right\},
\end{aligned}
$$

it follows that

$$
\sup \left\{\left\|d f_{p^{k}}^{k}(X)\right\|: X \in S\left(\mathfrak{g}_{-1}^{k}\right), p^{k} \in G^{k}\right\} \leq \lambda^{2} c_{k} .
$$

Since $f$ is not constant, $c_{k} \neq 0$, and now each map $f^{j}$ is bi-Lipschitz, and, by considering horizontal curves, we conclude that

$$
c_{k} \varrho(p, q) \leq \varrho(f(p), f(q)) \leq \lambda^{2} c_{k} \varrho(p, q) \quad \forall p, q \in G,
$$

as required. 
Remark 5.2. The argument above shows that if $f$ is defined in a domain $\Omega$ in $G$, then $f$ is locally a product mapping. Of course, this does not imply that $f$ is a product mapping, unless $\Omega$ is a product domain. However, if $f$ is 1-quasiconformal, then stronger conclusions do hold.

Corollary 5.3. Suppose that $G$ is a totally nonabelian Carnot group, with finest direct product decomposition $G^{1} \times \cdots \times G^{m}$, where $m>1$. Let $f: \Omega \rightarrow G$ be a 1quasiconformal map from a domain $\Omega$ in $G$ onto its image. Then $f$ is the restriction to $\Omega$ of the composition of a group automorphism that permutes the groups $G^{j}$ and a product affine map.

Proof. By [3], $f$ is smooth. By [4, Theorem 4.1], $f$ is an affine map. In particular, $f$ extends analytically to a conformal map on all of $G$. By Theorem 5.1 it follows that $f$ is is the composition of a group automorphism that permutes the groups $G^{j}$ and a product map.

Remark 5.4. We recall that if $G$ is the Heisenberg group $H^{n}$, then conformal maps on a domain in $G$ are restrictions of the action of an element of $S U(1, n+1)$ [12. However, if $G$ is the product of $m$ Heisenberg groups $H^{n_{l}}$ where $m \geq 2$, then most elements in $S U\left(1, n_{1}+1\right) \times \cdots \times S U\left(1, n_{m}+1\right)$ do not induce conformal maps on domains in $G$. Indeed, from the previous corollary, conformal maps are affine in this case.

\subsection{CR mappings on product groups.}

Theorem 5.5. Suppose that $G$ is a totally nonabelian Carnot group, with finest direct product decomposition $G^{1} \times \cdots \times G^{m}$, where $m>1$. Suppose that $G^{j}$ is tight when $j=1, \ldots, m$. Let $f: G \rightarrow G$ be a CR mapping. Then $f$ is the composition of a group automorphism that permutes the groups $G^{j}$ and a product CR mapping.

Proof. By Theorem 3.3, for tight Carnot groups CR and anti-CR diffeomorphisms are the same as conformal mappings. The conclusion now follows from Corollary 5.3.

\section{REFERENCES}

[1] Aldo Andreotti and C. Denson Hill, Complex characteristic coordinates and tangential Cauchy-Riemann equations, Ann. Scuola Norm. Sup. Pisa Cl. Sci. (3) 26 (1972), 299-324. MR460724

[2] Luca Capogna, Giovanna Citti, Enrico Le Donne, and Alessandro Ottazzi, Conformality and Q-harmonicity in sub-Riemannian manifolds, J. Math. Pures Appl. (9) 122 (2019), 67-124, DOI 10.1016/j.matpur.2017.12.006. MR3912638

[3] Luca Capogna and Michael Cowling, Conformality and Q-harmonicity in Carnot groups, Duke Math. J. 135 (2006), no. 3, 455-479, DOI 10.1215/S0012-7094-06-13532-9. MR2272973

[4] Michael G. Cowling and Alessandro Ottazzi, Conformal maps of Carnot groups, Ann. Acad. Sci. Fenn. Math. 40 (2015), no. 1, 203-213, DOI 10.5186/aasfm.2015.4008. MR3310079

[5] Michael G. Cowling and Alessandro Ottazzi, Structure of stratified groups I. Product decompositions, J. Lie Theory 27 (2017), no. 1, 177-183. MR.3521656

[6] C. Fefferman, A. Ionescu, T. Tao, and S. Wainger, Analysis and applications: The mathematical work of Elias Stein, Bull. Amer. Math. Soc. https://doi.org/10.1090/bull/1691, 2020.

[7] C. Robin Graham, On Sparling's characterization of Fefferman metrics, Amer. J. Math. 109 (1987), no. 5, 853-874, DOI 10.2307/2374491. MR.910354

[8] Marshall Hall Jr., A basis for free Lie rings and higher commutators in free groups, Proc. Amer. Math. Soc. 1 (1950), 575-581, DOI 10.2307/2032282. MR38336 
[9] C. Denson Hill and Mauro Nacinovich, Solvable Lie algebras and the embedding of CR manifolds (English, with Italian summary), Boll. Unione Mat. Ital. Sez. B Artic. Ric. Mat. (8) 2 (1999), no. 1, 121-126. MR1794546

[10] David Jerison and John M. Lee, The Yamabe problem on CR manifolds, J. Differential Geom. 25 (1987), no. 2, 167-197. MR880182

[11] David Jerison and John M. Lee, Intrinsic CR normal coordinates and the CR Yamabe problem, J. Differential Geom. 29 (1989), no. 2, 303-343. MR.982177

[12] A. Korányi and H. M. Reimann, Quasiconformal mappings on the Heisenberg group, Invent. Math. 80 (1985), no. 2, 309-338, DOI 10.1007/BF01388609. MR788413

[13] John M. Lee, The Fefferman metric and pseudo-Hermitian invariants, Trans. Amer. Math. Soc. 296 (1986), no. 1, 411-429, DOI 10.2307/2000582. MR837820

[14] John M. Lee, Pseudo-Einstein structures on CR manifolds, Amer. J. Math. 110 (1988), no. 1, 157-178, DOI 10.2307/2374543. MR926742

[15] V. S. Varadarajan, Lie groups, Lie algebras, and their representations, Prentice-Hall, Inc., Englewood Cliffs, N.J., 1974. Prentice-Hall Series in Modern Analysis. MR.0376938

[16] QingYan Wu and Wei Wang, Conformal mappings and CR mappings on the Engel group, Sci. China Ser. A 52 (2009), no. 12, 2759-2773, DOI 10.1007/s11425-009-0177-5. MR2577189

[17] Ts. Ya. U and V. Van, The Beltrami equations for quasiconformal mappings on strictly pseudoconvex hypersurfaces (Russian, with Russian summary), Sibirsk. Mat. Zh. 53 (2012), no. 2, 396-417, DOI 10.1134/S0037446612020140; English transl., Sib. Math. J. 53 (2012), no. 2, 316-334. MR2975944

[18] Q. Y. Wu and Z. W. Fu, 1-Quasiconformal Mappings and CR Mappings on Goursat Groups, Sci. World J., Volume 2014, Article ID 930571, 9 pages.

[19] Xiangdong Xie, Quasisymmetric homeomorphisms on reducible Carnot groups, Pacific J. Math. 265 (2013), no. 1, 113-122, DOI 10.2140/pjm.2013.265.113. MR.3095115

School of Mathematics and Statistics, University of New South Wales, UnSW SydNEY 2052, Australia

Email address: m.cowling@unsw.edu.au

Department of Mathematics, Macquarie University NSW 2109, Australia

Email address: ji.li@mq.edu.au

School of Mathematics and Statistics, University of New South Wales, UNSW SydNEY 2052, Australia

Email address: a.ottazzi@unsw.edu.au

Department of Mathematics, Linyi University, Shandong, 276005, People's Republic OF CHINA

Email address: wuqingyan@lyu.edu.cn 